Check for updates

Cite this: RSC Adv., 2018, 8, 37623

\title{
Synthesis of novel cyclodextrin-modified reduced graphene oxide composites by a simple hydrothermal method $\uparrow$
}

\author{
Qingli Huang, ${ }^{\text {ab }}$ MingYan Li, ${ }^{\mathrm{a}}$ LiLi Wang, ${ }^{\mathrm{b}}$ Honghua Yuan, ${ }^{\mathrm{b}}$ Meng Wang, \\ Yongping $\mathrm{Wu}\left(\mathbb{D}{ }^{* a}\right.$ and Ting $\mathrm{Li}^{* \mathrm{~b}}$
}

Cyclodextrin ( $\beta-C D)$-functionalized reduced graphene oxide was successfully synthesized by a simple hydrothermal method, followed by conjugating with polyethylene glycol (PEG) and folic acid (FA). Microscopic and spectroscopic techniques were used to characterize the nanocomposites. Photothermal experiments showed that $\beta$-CD-functionalized reduced graphene oxide exhibited higher photothermal conversion efficiency in the near infrared region than reduced graphene oxide functionalized with other molecules under the same conditions. Cytotoxicity experiments indicated that rGO@CD@PEG@FA possessed good biocompatibility even at high concentration. When doxorubicin (DOX) was loaded on the rGO@CD@PEG@FA nanocomposite, it showed the stimulative effect of heat, $\mathrm{pH}$ response, and sustained drug release. Cytotoxicity experiments also confirmed the targeted effect and high efficiency of the combined therapy. The findings of the present study provide an ideal drug delivery system for malignant cancer therapy due to the advanced synergistic chemo-photothermal targeted therapy and good drug release properties.

Received 20th September 2018
Accepted 19th October 2018

DOI: $10.1039 / c 8 r a 07807 f$

rsc.li/rsc-advances oxide (GO) and reduced graphene oxide (rGO) and render new physicochemical and biological advantages to the bare GO or rGO. ${ }^{16-19}$ Cyclodextrins (CDs), as biodegradable polymers, could modify the NPs' behavior at the biointerfaces and improve control over drug release. ${ }^{20-23}$ There are several advantages: (i) CD itself has good ability to load drugs; (ii) CDs can work as capping molecules to cover the GO carriers and protect the loaded compounds from the external environment; (iii) CDs have good biocompatibility, facilitating the safe and efficient delivery of drugs; and (iv) CDs have many active groups, which could be modified further by other molecules. Owing to these advantages, CDs have been reported for the delivery of several therapeutics. ${ }^{24-27}$

As a traditional therapy method, chemotherapy sometimes does not achieve the ideal therapeutic effect. Photothermal therapy (PTT), as a minimally invasive therapeutic methodology, has attracted much attention as a promising supplement to traditional chemotherapy. ${ }^{28}$ The development of the nearinfrared (NIR) laser-induced PTT depends on gaining access to biocompatible and efficient photothermal coupling agents. As well-known NIR photothermal conversion agents, GO and rGO have been widely investigated due to their strong lightabsorbing capability and encouraging photothermal therapeutic effects in both in vitro and in vivo experiments. ${ }^{28-37}$ Though some progress has been achieved, the development of methods to synthesize GO (rGO)-based therapeutic systems is necessary. In addition, during cancer therapy, suitable targeting of tumor cells is very important because non-specific targeting

\footnotetext{
${ }^{a}$ Department of Pathology, Laboratory of Clinical and Experimental Pathology, Xuzhou Medical University, No. 209 Tongshan Road, Xuzhou, Jiangsu, 221004, China

${ }^{b}$ Research Facility Center for Morphology of Xuzhou Medical University, No. 209 Tongshan Road, Xuzhou, Jiangsu, 221004, China

$\dagger$ Electronic supplementary information (ESI) available. See DOI: 10.1039/c8ra07807f
} 
can lead to destruction of normal cells. Lack of suitable targeting to tumor cells seriously confines rGO's therapeutic applications. For ovarian cancer, the folate receptors are overexpressed on the surface of the cancer cells. Folic acid (FA) molecules could thus be used to target these cells. ${ }^{38-43}$

Herein, we report the rational design and successful synthesis of rGO@CD@PEG@FA nanocomposites by a simple hydrothermal method. The as-prepared rGO@CD@PEG@FA nanocomposites show low cytotoxicity and excellent photothermal conversion in vitro, which is promising for photothermal therapy (PPT) against cancer. When the anticancer drug doxorubicin (DOX) is loaded into rGO@CD@PEG@FA nanocomposites, it is effectively delivered to target ovarian cancer cells with a pH-sensitive release profile and thus employed for chemotherapy of cancer cells. Importantly, the combination of PPT and chemotherapy demonstrates better therapy effects on cancer cells than the individual therapy approaches in vitro.

\section{Experimental section}

\subsection{Material}

Graphene oxide (GO) was obtained from XFNANO Co., Ltd. (Nanjing, China). Doxorubicin hydrochloride (DOX), (3-aminopropyl)triethoxysilane (APTES), carbodiimide (EDC), $\mathrm{N}$ hydroxysuccinimide (NHS) and folic acid (FA) were purchased from Aladdin Reagent Co., Ltd. (Shanghai, China). 6-Amino-6deoxy- $\beta$-cyclodextrin (CD) was acquired from Zhiyuan Biotechnology Co., Ltd. (Binzhou, China). Dulbecco's modified Eagle medium (DMEM), penicillin-streptomycin, and fetal calf serum (FBS) were purchased from Kaiji Biotechnology Co., Ltd. (Nanjing, China). Cell counting kit-8 (CCK-8) was purchased from VICMED Biotechnology Co., Ltd. (Xuzhou, China). Carboxymethyl-PEG-carboxymethyl (MW 10 000) was obtained from Yare Biotechnology Co., Ltd. (Shanghai, China). All chemicals are analytically pure and were used as received without further purification. Deionized water was used throughout the experiment.

\subsection{Methods}

2.2.1 Synthesis of the rGO@CD. $100 \mathrm{mg}$ of 6-amino-6deoxy- $\beta$-cyclodextrin (CD) was added to the GO suspension with $24 \mathrm{~mL}$ water $\left(1 \mathrm{mg} \mathrm{mL}^{-1}\right)$, respectively, and stirred continuously for $1 \mathrm{~h}$ at room temperature. Then, the mixed solution was transferred into a Teflon-lined autoclave of $30 \mathrm{~mL}$ capacity. After being sealed and heated at $100{ }^{\circ} \mathrm{C}$ for $12 \mathrm{~h}$, the autoclave was cooled to room temperature naturally. The resulting products were collected by centrifugation, washed with distilled water and ethanol several times, and finally dried. rGO modified by different molecules [1 $\mathrm{mL}$ (3-aminopropyl)triethoxysilane (APTES) and $100 \mathrm{mg}$ albumin from bovine serum (BSA)] were also prepared using the same procedure.

\subsubsection{Synthesis of the rGO@CD@PEG}

$10 \mathrm{mg}$ rGO@CD was dispersed in $10 \mathrm{~mL}$ deionized water by ultrasonication. Then, $20 \mathrm{mg}$ carboxymethyl-PEG- carboxymethyl was added into the above solution and stirred at room temperature for $12 \mathrm{~h}$. The resulting products were collected by centrifugation, washed with distilled water and ethanol, and finally stored in $10 \mathrm{~mL}$ deionized water.

\subsubsection{Preparation of the rGO@CD@PEG@FA}

Briefly, $13 \mathrm{mg}$ folic acid was dispersed in $12 \mathrm{~mL}$ PBS solution by simple ultrasonication. Then, $30 \mathrm{mg}$ of 1-ethyl-3-(3(dimethylamino)propyl)-carbodiimide (EDC) and $50 \mathrm{mg}$ of $\mathrm{N}$ hydroxysuccinimide (NHS) were added into the solution, and the reaction mixture was allowed to stir for $6 \mathrm{~h}$ in the dark. Thus, the activated folic acid solution was obtained. Finally, $8 \mathrm{~mL}$ rGO@CD@PEG solution was added dropwise to $4 \mathrm{~mL}$ activated folic acid solution. The reaction mixture was allowed to stir overnight in the dark. Further dialysis was carried out using molecular weight cut-off membrane (3500 Da, MWCO) to remove unreacted molecules $(<3000 \mathrm{MW})$ such as NHS, EDC and unreacted FA.

\subsubsection{DOX loading and release}

$5 \mathrm{~mL}$ DOX solution ( $2 \mathrm{mg} \mathrm{mL}{ }^{-1}$ ) was added to $5 \mathrm{~mL}$ of the asprepared rGO@CD@PEG@FA solution (2 mg mL $\left.{ }^{-1}\right)$ under vigorous stirring for $12 \mathrm{~h}$ in the dark. Unbound DOX particles were removed by ultracentrifugation at $12000 \mathrm{rpm}$. The unloaded DOX concentration and DOX loading amount were calculated from a calibration curve of several DOX solutions with known concentrations using a Mode 680 Microplate Reader (BIO-RAD, USA). The release of DOX was tested in phosphate-buffered solution with different $\mathrm{pH}$ levels at $37^{\circ} \mathrm{C}$. The mixture was centrifuged a certain number of times to measure the absorbance of the supernatant and estimate the released amount. For NIR laser irradiation, the samples were irradiated by $808 \mathrm{~nm}$ laser ( $2 \mathrm{~W}$, LWIRL808-10W-F) for $10 \mathrm{~min}$ at a certain time interval.

\subsection{Characterization}

TEM images of the as-prepared samples were obtained by a transmission electron microscopy (FEI Tecnai 12). FTIR spectra were recorded in the transmittance mode in the range of $400-4000 \mathrm{~cm}^{-1}$ at using a Cary $610 / 670$ spectrometer (Varian, USA). The as-prepared samples were thoroughly milled with $\mathrm{KBr}$ into a pellet before the measurement. UV-vis absorption spectra were recorded on a Shimadzu UV-2550 spectrophotometer in the range of $200-800 \mathrm{~cm}^{-1}$. The Raman spectra were obtained on a Renishaw Invia Raman spectrometer using $532 \mathrm{~nm}$ laser at room temperature in the range of $2000-400 \mathrm{~cm}^{-1}$. An $808 \mathrm{~nm}$ laser (LWIRL808-10W-F, Laserwave Co.) was utilized as a selected NIR light to irradiate rGO with different aqueous dispersions of modifying molecules $\left(3 \mathrm{~mL}, 100 \mu \mathrm{g} \mathrm{mL} \mathrm{m}^{-1}\right)$ to induce photothermal effect. Cytotoxicity assays were conducted on a microplate reader (Bio-rad, USA) according to a standard CCK-8 method. The relative cell viability was measured by comparison with the control well containing only the cells. 


\subsection{Cell culture and viability assay}

2.4.1 Cell culture. Ovarian cancer cells (SKOV3) were cultured in Dulbecco's modified Eagle medium (DMEM) supplemented with $10 \%$ FBS and $1 \%(\mathrm{v} / \mathrm{v})$ penicillin-streptomycin. Cells were incubated for 3 days in a humidified $5 \% \mathrm{CO}_{2}$ containing balanced air at $37{ }^{\circ} \mathrm{C}$. The media were changed every day.

2.4.2 Cell viability assay. Cytotoxicity assays were conducted according to a standard CCK- 8 method. $200 \mu \mathrm{L}$ of the cultured cells, at a density of $2 \times 10^{4}$ cells per $\mathrm{mL}$, was placed in each well of a 96-well plate. Then, the cells were incubated at $37{ }^{\circ} \mathrm{C}$ in $5 \% \mathrm{CO}_{2}$ atmosphere for $24 \mathrm{~h}$. The medium was removed, and cells were then treated with the medium containing as-prepared samples for $24 \mathrm{~h}$ at $37^{\circ} \mathrm{C}$ in an atmosphere of $5 \% \mathrm{CO}_{2}$. Finally, $10 \mu \mathrm{L}$ of CCK- 8 was added into each well, and all cells were incubated for another $2 \mathrm{~h}$. The absorbance of the solution at $450 \mathrm{~nm}$ was measured using a microplate reader. The relative cell viability was measured by comparing the control well containing only the cells.

\section{Results and discussion}

The morphology of the as-prepared samples is revealed by the transmission electron microscope (TEM) images in Fig. 1. It can be found that GO is relatively transparent, and its surface is smooth in Fig. 1a. After it was functionalized with CD (Fig. 1b), the product became rougher and thicker than GO, which reflects that the $\mathrm{CD}$ was grafted successfully on the surface of GO.For rGO@CD@PEG and rGO@CD@PEG@FA, it is difficult to observe an obvious change in morphology due to the influence of $\mathrm{CD}$ on the surface of rGO. The TEM images of rGO@CD@PEG@FA in PBS at pH 5.0 and 7.4 after 6 days are shown in Fig. S1. $\dagger$ No significant morphology and size changes were observed, indicating that rGO@CD@PEG@FA possesses good stability.

FTIR was also carried out to investigate the formation of rGO@CD@PEG@FA. For the pure GO spectrum in Fig. 2a, the characteristic peaks of GO at 3529, 1735, and $1090 \mathrm{~cm}^{-1}$ are ascribed to the stretching vibrations of $\mathrm{O}-\mathrm{H}, \mathrm{C}-\mathrm{O}$ and $\mathrm{C}=\mathrm{O}$ in the carbonyl and carboxylic groups, respectively. ${ }^{44}$ However, in the spectrum of rGO@CD (Fig. 2b), the characteristic absorption bands of oxygen-containing groups decreased dramatically,

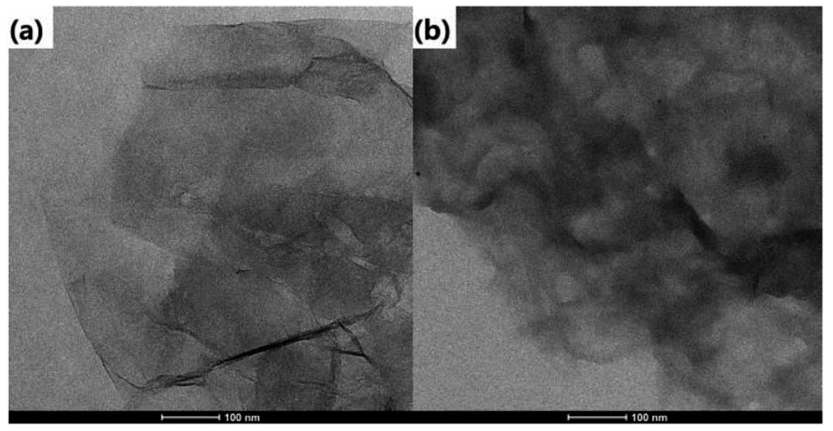

Fig. 1 TEM images of (a) GO and (b) rGO@CD.

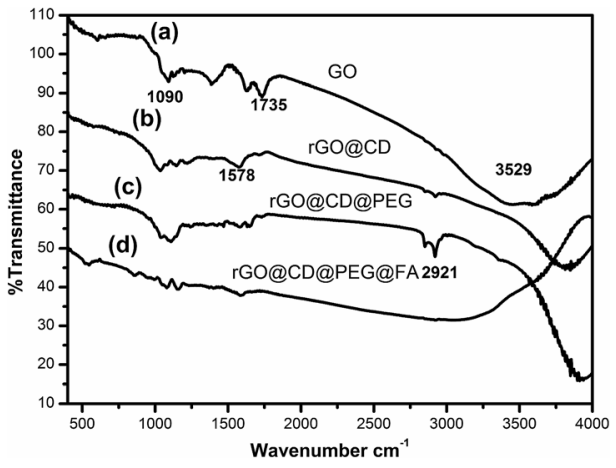

Fig. 2 FTIR spectra of the prepared samples: (a) GO, (b) rGO@CD, (c) rGO@CD@PEG, (d) rGO@CD@PEG@FA.

indicating that GO has been reduced to rGO. A new peak at $1578 \mathrm{~cm}^{-1}$ was found, which can be attributed to the $\mathrm{N}-\mathrm{H}$ stretching vibration of 6-amino-6-deoxy-beta-cyclodextrin (CD). ${ }^{45}$ With the appearance of the strong vibrations of methylene groups of PEG chains at $2921 \mathrm{~cm}^{-1}$ in Fig. 2c, it is clearly demonstrated that PEG had been conjugated on the rGO@CD successfully. ${ }^{46}$ In Fig. 2d, a broad peak from $3000-3500 \mathrm{~cm}^{-1}$ $\left(-\mathrm{COOH}\right.$ and $\left.-\mathrm{NH}_{2}\right)$ appeared, indicating the existing of FA on the composites.

UV-vis spectroscopic analysis was also used to ascertain the modification process of rGO. As shown in Fig. 3, a typical peak found at $230 \mathrm{~nm}$ corresponds to the $\pi-\pi^{*}$ transitions of the aromatic $\mathrm{C}=\mathrm{C}$ bonds, and a weak shoulder peak at $300 \mathrm{~nm}$ is due to $n-\pi^{*}$ transitions of $\mathrm{C}=\mathrm{O}$ bonds. ${ }^{47}$ After modification and reduction by $\mathrm{CD}$, a broad peak showed up around $266 \mathrm{~nm}$, suggesting the reduction and modification of $\mathrm{GO}$ by $\mathrm{CD}^{47}$ However, there is little change in the absorption spectrum of rGO@CD@PEG due to no obvious absorption of PEG. For GO@CD@PEG@FA, a strong characteristic peak for folic acid at $282 \mathrm{~nm}$ appeared after grafting FA. $^{48}$

Raman spectroscopy was also used to characterize the structure of GO in Fig. 4. The Raman spectrum has two prominent peaks at $1598 \mathrm{~cm}^{-1}$ and $1350 \mathrm{~cm}^{-1}$ corresponding to the well documented $\mathrm{G}$ and $\mathrm{D}$ bands. The $\mathrm{G}$ band is assigned to the stretching vibration of $\mathrm{sp}^{2}$-bonded carbon atoms, and the $\mathrm{D}$ band corresponds to the vibrations of carbon atoms with the $\mathrm{sp}^{3}$ electronic configuration of disordered graphene. ${ }^{49}$ After

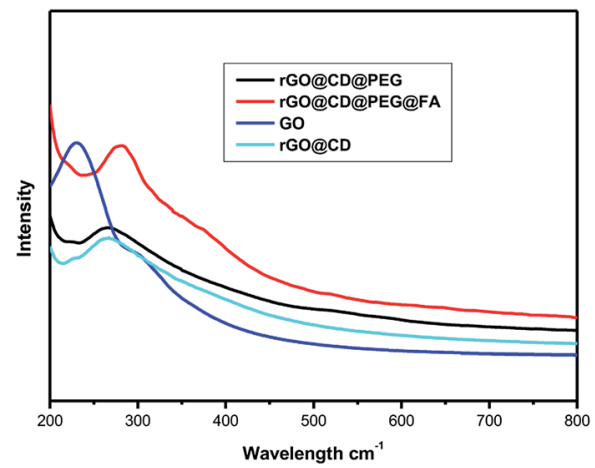

Fig. 3 UV-vis spectra of the prepared samples. 


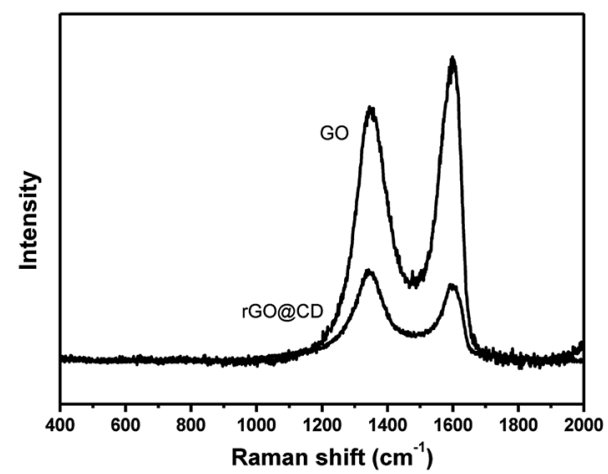

Fig. 4 Raman spectra of the prepared samples.

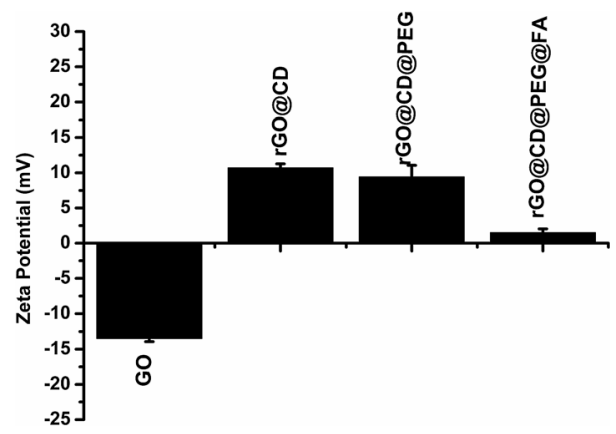

Fig. 5 Zeta potential of the prepared samples.

functionalizing with $\mathrm{CD}$, the intensity ratio of the $\mathrm{D}$ and $\mathrm{G}$ bands of the product obviously increased in comparison with those of the original graphene oxide in the same test conditions, indicating the reduction of GO by CD. ${ }^{50}$

Moreover, the zeta potential of the obtained samples after each of the coating steps was also measured to monitor and verify the successful modification of $\beta$-CD, PEG and FA. As could be observed in Fig. 5, the zeta potential of GO was negative. After modification with $\beta$-CD, the composites showed positive zeta potential. Upon further modification with PEG and FA, there is an obvious change in zeta potential, indicating the composition change on the surface of rGO.

Graphene and graphene composites have good photothermal conversion performance and could be used as photothermal agents for treating cancer under NIR irradiation. The photothermal performance of the as-prepared samples was investigated in their aqueous dispersion $\left(100 \mu \mathrm{g} \mathrm{mL} \mathrm{m}^{-1}\right)$. Each sample was exposed to the same NIR irradiation $(808 \mathrm{~nm}$, continuous wave, $1 \mathrm{~W}, 300 \mathrm{~s}$ ), and the temperature change was recorded using a temperature controller, model $\mathrm{CH} 702$. The rGO@CD showed excellent photothermal performance due to the modification of CD. In Fig. 6a, we can find that the temperature difference of the rGO@CD aqueous dispersion (about $18{ }^{\circ} \mathrm{C}$ ) is larger than that of GO aqueous dispersion (about $10^{\circ} \mathrm{C}$ ) and deionized water (about $4{ }^{\circ} \mathrm{C}$ ) with NIR irradiation for $300 \mathrm{~s}$. It is obvious that an incremental amount of photothermal conversion effect was achieved when CD was modified on the surface of GO. To confirm the effect of $\mathrm{CD}$, the photothermal performance of rGO functionalized with other
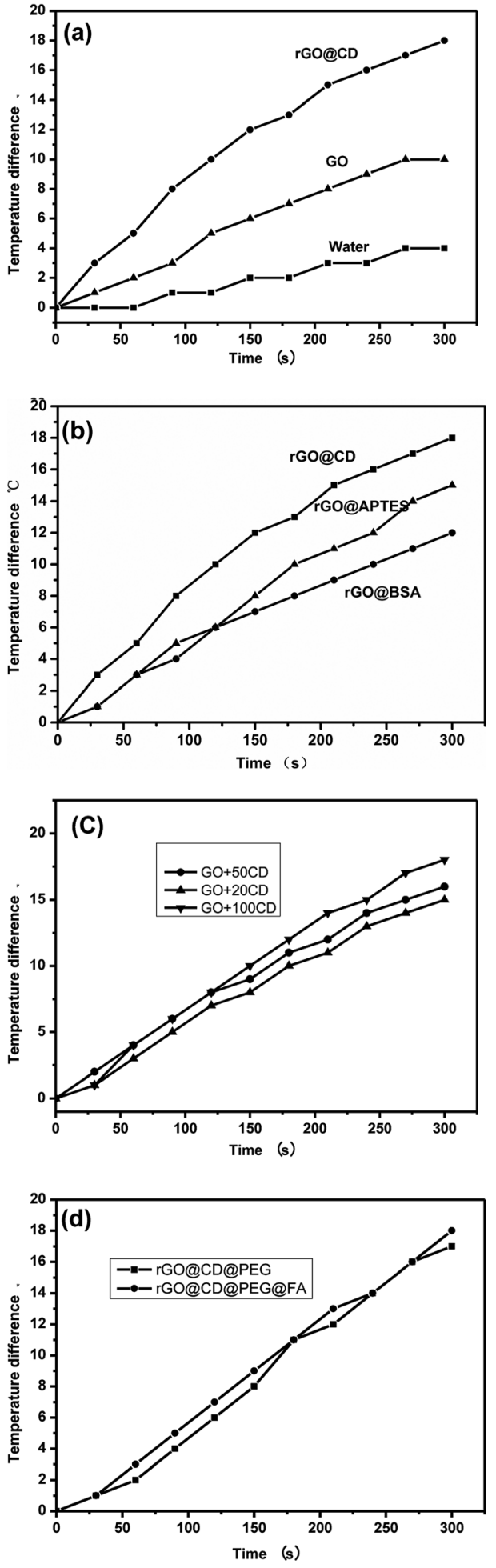

Fig. 6 The photothermal performance of samples under the same NIR irradiation condition ( $808 \mathrm{~nm}$, continuous wave, $1 \mathrm{~W}, 300 \mathrm{~s}$ ): (a) pure water, GO and rGO@CD; (b) rGO functionalized with different molecules (CD, APTES and BSA); (c) the products prepared with different amounts of $C D$; (d) the rGO@CD further modified with PEG and FA.

molecules (APTES and BSA) was also investigated under the same conditions. Fig. $6 \mathrm{~b}$ shows the temperature difference of these molecules after NIR irradiation under the same conditions. It can be found that the rGO@CD had the best 
photothermal efficiency among all samples. This may be due to the differences in the structure of CD, APTES and BSA. The better photothermal performance of the rGO@CD than the rGO-APTES and rGO-BSA can be assigned to the unique structure of CD, with its cavity structure. ${ }^{51,52}$ The detailed mechanism needs further investigation. The photothermal conversion efficiency of rGO@CD was also calculated to be $46 \%$ according to previous literature $^{53-55}$ (Fig. S2-S4 in ESI $\dagger$ ). In addition, the amount of CD on the surface of rGO also determined its photothermal performance. Fig. 6c shows the photothermal performance of rGO@CD with different amounts of CD $(20 \mathrm{mg}$, $50 \mathrm{mg}$ and $100 \mathrm{mg}$ ) after NIR irradiation under the same condition. The results showed that more CD contributed higher photothermal performance, which further accounts for the validity of $\mathrm{CD}$. However, $\mathrm{CD}$ residue was found outside $\mathrm{rGO}$ when the amount of CD was larger than $100 \mathrm{mg}$. Herein, in our work, the appropriate amount of CD is $100 \mathrm{mg}$. At the same time, there is little influence on its photothermal performance when further modified with PEG and FA (Fig. 6d).

As a matter of fact, graphene and graphene composites are good candidates for loading and delivery of the anticancer drug DOX. Fluorescence spectra were used to ascertain the conjugation of DOX onto rGO@CD@PEG@FA@DOX. Fig. 7 represents the fluorescence spectra of all samples. Before DOX was loaded, no fluorescence signals were found for the samples (GO, rGO@CD, rGO@CD@PEG and rGO@CD@PEG@FA).However, after DOX was introduced, the appearance of the peak at $602 \mathrm{~nm}$ in rGO@CD@PEG@FA@DOX, which is characteristic for the pure DOX, confirmed the successful loading of DOX. The loading amount of DOX is $0.91 \mathrm{mg} \mathrm{mg}^{-1}$ at the neutral condition (pH 7.4) in our work. DOX has different prototropic forms at different $\mathrm{pH}$ values, owing to the presence of hydroxyl, carbonyl and amino groups on the sugar sidechain. ${ }^{56}$ The primary amino groups of DOX molecules are mostly protonated when $\mathrm{pH}$ is 7.4. ${ }^{57}$ As a hydrochloride salt, DOX can interact with $\beta$-CD in a $1: 1$ stoichiometry by hydrogen bonding and hydrophobic interactions in phosphate-buffered saline solution, resulting in an inclusion complex. The formation constant $\left(K_{\mathrm{f}}\right)$ was reported to be $210 \mathrm{M}^{-1}\left(25^{\circ} \mathrm{C}\right) .{ }^{56}$ In addition, the protonated form of DOX can also interact with the negative surface of GO by electrostatic interaction and hydrogen bonding. ${ }^{58}$ Meanwhile, intracellular DOX release was also studied by cell fluorescence imaging (Fig S5 $\dagger$ ). Red fluorescence was observed from DOX. Uniform bright red fluorescence in the SKOV3 cell could be observed, which was due to the release of fluorescent DOX entering the cell.

The release profile of DOX from rGO@CD@PEG@FA@DOX was studied at $\mathrm{pH} 7.4$ (corresponding to the environment of blood) and 5.0 (simulating the $\mathrm{pH}$ in mature endosomes of tumor cells) at a temperature of $37^{\circ} \mathrm{C}$, respectively. As shown in Fig. 8 (labels a and b), the rate of drug release was predominantly in a pH-dependent manner, with a sustained profile. DOX was released continuously over $120 \mathrm{~h}$, and $33.7 \%$ and $41.9 \%$ of total DOX was released at $\mathrm{pH}=7.4$ and 5.0 , respectively. Noticeably, the drug release was rapid in the case of an acidic environment (tumor cell environment), which is consistent with early reports. ${ }^{17,39}$ In addition, we found that the NIR

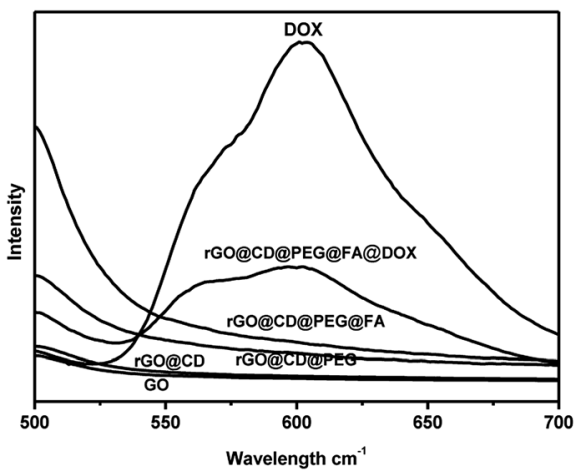

Fig. 7 Fluorescence spectra of the as-prepared samples.

laser irradiation could promote the release of DOX at $\mathrm{pH}=5.0$. About $52.2 \%$ of total DOX was released at $\mathrm{pH}=5.0$ under NIR laser irradiation, shown in Fig. 8(c). The cumulative heat destroys the electrostatic interaction between DOX and rGO, which significantly increases the release of DOX and the sensitivity of chemotherapy. As a result, rGO@CD@PEG@FA@DOX can be considered to be unique pH-sensitive and NIRstimulated cancer therapeutic agent, which has potential to reduce the side effects and enhance the therapeutic effect.

DOX is used widely in chemotherapy for cancer. However, current chemotherapies have many well-known disadvantages, including systemic side effects, relatively poor specificity toward malignant tissues, drug resistance and low efficacy. It was previously demonstrated that photo-thermal therapy (PTT) could lead to precise targeted treatment at the specific site, which is considerably less invasive than surgery. GO and rGO can produce a large amount of active oxygen species (ROS) and could be used as PTT agents for cancer. Consequently, it was highly expected that the synthesized rGO@CD@PEG@FA@DOX possesses both chemotherapeutic and PTT properties. For cancer therapeutic agents, safety and in vitro cytocompatibility are essential. To show the safety and in vitro cytocompatibility of rGO@CD@PEG@FA, the ovarian cancer cells (SKOV3) were incubated for $24 \mathrm{~h}$ with different concentrations of the asprepared samples $\left(25 \mu \mathrm{g} \mathrm{mL}{ }^{-1}, 50 \mu \mathrm{gL}^{-1}\right.$ and $100 \mu \mathrm{g} \mathrm{mL}{ }^{-1}$, see Fig. 9). Compared to viability of cells with pure GO, higher viability of cells was found when they were incubated with rGO@CD@PEG@FA (Fig. 9a). Fig. 9b shows the anticancer

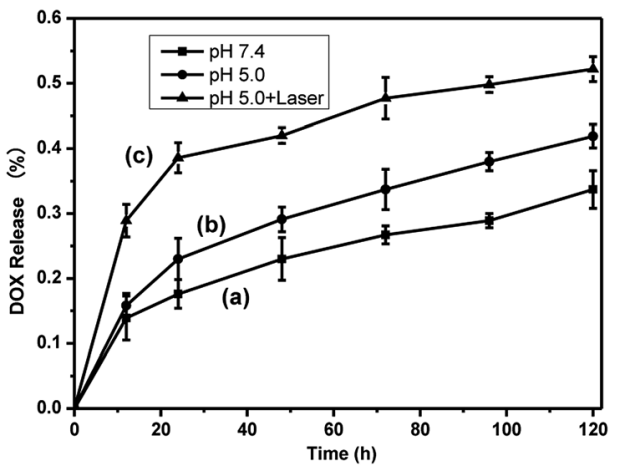

Fig. 8 DOX release profile (a) at $\mathrm{pH}=7.4$, (b) without and (c) with laser irradiation at $\mathrm{pH}=5.0$ over $120 \mathrm{~h}$. 

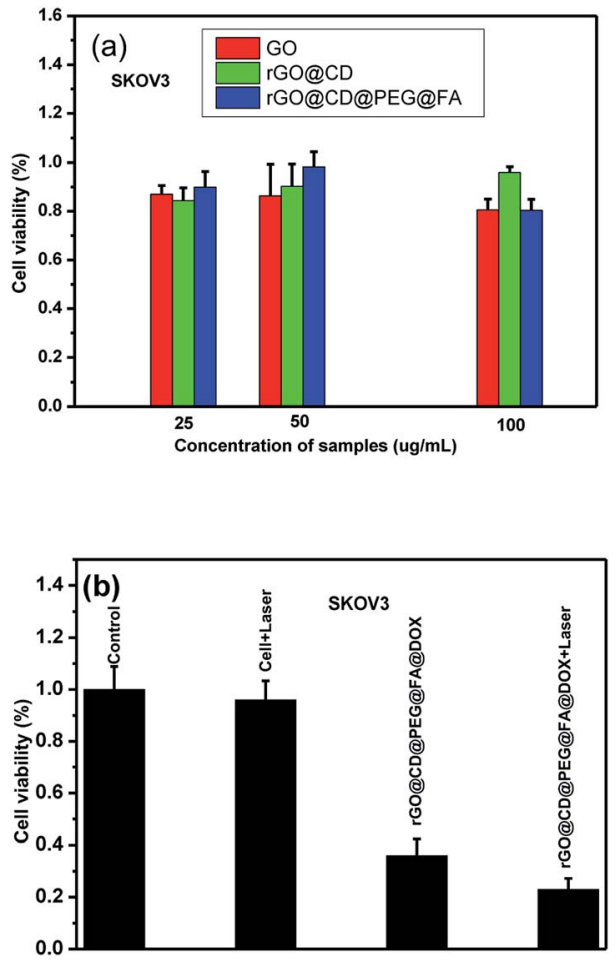

Fig. 9 (a) Viability of SKOV3 cells incubated with different samples at different concentrations. (b) Viability of SKOV3 cells incubated with $100 \mu \mathrm{g} \mathrm{mL}^{-1}$ rGO@CDAPEG@FA@DOX for $24 \mathrm{~h}$ without and with NIR laser irradiation at low power density $\left(600 \mathrm{~mW} \mathrm{~cm}^{-2}\right.$ for $\left.5 \mathrm{~min}\right)$.

performance of rGO@CD@PEG@FA@DOX by chemotherapy and synergistic chemo-photothermal therapy methods. There was almost no change for the pure medium group under NIR laser irradiation. The cell viability is lower with synergistic chemo-photothermal therapy (23\%) than with single chemotherapy (36\%). This means the $808 \mathrm{~nm}$ NIR effectively activated rGO@CD@PEG@FA@DOX and had a lethal effect on cancer cells. The as-prepared rGO@CD@PEG@FA@DOX is promising as a cancer therapeutic agent via synergistic chemophotothermal therapy, and its possible mechanism is discussed. ${ }^{5-63}$ On one hand, the intrinsic high optical absorption of rGO composites in the near-infrared (NIR) region elevated the temperature of tumor sites, generating many ROS such as $\cdot \mathrm{OH}$, $\mathrm{O}^{2-\cdot}$ and $\mathrm{H}_{2} \mathrm{O}_{2}$. These $\cdot \mathrm{OH}, \mathrm{O}^{2-\cdot}$ and $\mathrm{H}_{2} \mathrm{O}_{2}$ can damage and/or inhibit proteins in several ways-one is the direct oxidation of amino acids by ROS. Thus, photothermal ablation of tumors occurred. On the other hand, the heat generated by photothermal effect could destroy the electrostatic interaction between DOX and GO composites, which significantly increases the release of DOX and the cells' sensitivity to chemotherapy. Furthermore, the photochemical internalization effect, which is related to site-specific release of chemotherapeutics inside the cells, led to disintegration of endosomes and lysosomes.

Different from the high expression level of folate receptor in ovarian cancer cells, the folate receptor expression level is very low in prostate cancer cells (DU145). Finally, the targeting ability of rGO@CD@PEG@FA@DOX for ovarian cancer cells was evaluated by the introduction of prostate cancer cells

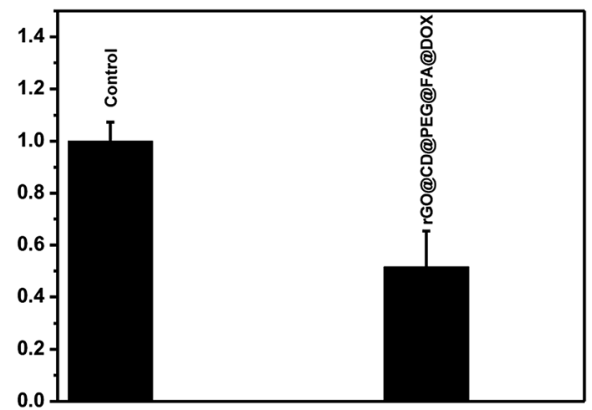

Fig. 10 Viability of DU145 cells incubated without and with $100 \mu \mathrm{g}$ $\mathrm{mL}^{-1}$ rGO@CD@PEG@FA@DOX for $24 \mathrm{~h}$.

(DU145) in Fig. 10. It can be seen that rGO@CD@PEG@FA@DOX exhibited significantly higher cytotoxicity in ovarian cancer cells (36\% viability, Fig. 9) than that in prostate cancer cells (51\% viability, Fig. 10$)$. This could be due to the different expression level of folate receptor in different cells. The same phenomenon was observed in early works, which demonstrated that folate could significantly enhance the cellular uptake of the drug delivery system in cells with high expression levels of folate receptor. ${ }^{39-43}$

\section{Conclusion}

In summary, we have designed and synthesized a rGO@CD@PEG@FA nanocomposite as a new nanohybrid drug carrier and photothermal therapeutic agent. Various spectroscopic and microscopic techniques were used to characterize this nanocomposite. The results show that CD, PEG and FA were successfully conjugated on the surface of rGO. The as-prepared rGO@CD@PEG@FA nanocomposite not only showed good pHtriggered release of DOX but also had good targeting ability. Furthermore, this nanocomposite showed photo-thermal conversion properties under NIR irradiation. Based on the present findings, the rGO@CD@PEG@FA nanocomposite has the potential for tumor-targeted release of drugs and heat, facilitating targeted cancer chemo-photothermal therapy in a single system.

\section{Conflicts of interest}

There are no conflicts to declare.

\section{Acknowledgements}

This work is funded by the National Natural Science Foundation of China (No. 81372479 and 21505118), Natural Science Foundation of Jiangsu Province of China (BK 20150438) and Postdoctoral Research Funding Program of Jiangsu Province of China (1701133C).

\section{References}

1 H. Ye, A. A. Karim and X. J. Loh, Mater. Sci. Eng., C, 2014, 45, 609-619. 
2 Z. Y. Xu, S. Wang, Y. J. Li, M. W. Wang, P. Shi and X. Y. Huang, ACS Appl. Mater. Interfaces, 2014, 6, 1726817276.

3 S. C. Mohanta, A. Saha and P. S. Devi, Mater. Today, 2018, 5, 9715-9725.

4 N. Cristina, V. E. Sergio and d. P. C. Maria, J. Biol. Inorg Chem., 2018, 23, 331-345.

5 R. Lima-Sousa, D. Melo-Diogo, C. G. Alves, E. C. Costa, P. Ferreira, R. O. Louro and I. J. Correia, Carbohydr. Polym., 2018, 200, 93-99.

6 D. P. Singh, C. E. Herrera, B. Singh, S. Singh, R. K. Singh and R. Kumar, Mater. Sci. Eng., C, 2018, 86, 173-197.

7 M. Orecchioni, R. Cabizza, A. Bianco and L. G. Delogu, Theranostics, 2015, 5, 710-723.

8 Z. Li, A. L. B. de Barros, D. C. F. Soares, S. N. Moss and L. Alisaraie, Int. J. Pharm., 2017, 524, 41-54.

9 S. Laksee, S. Puthong, P. Kongkavitoon, T. Palaga and N. Muangsin, Carbohydr. Polym., 2018, 198, 495-508.

10 G. Paramasivam, N. Kayambu, A. M. Rabel, A. K. Sundramoorthy and A. Sundaramurthy, Acta Biomater., 2017, 49, 45-65.

11 Y. Liu, H. Zhong, Y. Qin, Y. Zhang, X. F. Liu and T. Zhang, RSC Adv., 2016, 6, 30184-30193.

12 J. H. Zhu, M. J. Chen, Q. L. He, L. Shao, S. Y. Wei and Z. H. Guo, RSC Adv., 2013, 3, 22790-22824.

13 Y. Pan, N. G. Sahoo and L. Li, Expert Opin. Drug Delivery, 2012, 9, 1365-1376.

14 S. Augustine, J. Singh, M. Srivastava, M. Sharma, A. Das and B. D. Malhotra, Biomater. Sci., 2017, 5, 901-952.

15 X. L. Wang and G. Q. Shi, Phys. Chem. Chem. Phys., 2015, 17, 28484-28504.

16 C. Wang, Z. Q. Zhang, B. B. Chen, L. Q. Gu, Y. Li and S. W. Yu, J. Colloid Interface Sci., 2018, 516, 332-341.

17 R. Wang, D. Shou, O. Lv, Y. Kong, L. H. Deng and J. Shen, Int. J. Biol. Macromol., 2017, 103, 248-253.

18 S. S. Mu, G. W. Li, Y. Y. Liang, T. Wu and D. Ma, Mater. Sci. Eng., C, 2017, 78, 639-646.

19 Y. T. Fan, Q. Y. Sun, H. Gu, W. X. Li, Y. Y. Chen, J. Wang, N. L. Zhou and Y. H. Xiao, Nanotechnology, 2014, 25, 255601.

20 N. Zafar, H. Fessi and A. Elaissari, Int. J. Pharm., 2014, 461, 351-366.

21 S. Bai, M. L. Hou, X. X. Shi, J. C. Chen, X. Q. Ma, Y.-E. Gao, Y. J. Wang, P. Xue, Y. J. Kang and Z. G. Xu, Carbohydr. Polym., 2018, 193, 153-162.

22 T. Loftsson, P. Jarho, M. Masson and T. Jarvinen, Expert Opin. Drug Delivery, 2005, 2, 335-351.

23 S. Lu, A. Wang, Y. J. Ma, H. Y. Xuan, B. Zhao, X. D. Li, J. H. Zhou, L. Zhou and S. H. We, Carbohydr. Polym., 2016, 148, 236-242.

24 G. C. Wei, R. H. Dong, D. Wang, L. Feng, S. L. Dong, A. X. Song and J. C. Hao, New J. Chem., 2014, 38, 140-145.

25 E. Einafshar, A. H. Asl, A. H. Nia, M. Mohammadi, A. Malekzadeh and M. Ramezani, Carbohydr. Polym., 2018, 194, 103-110.

26 N. Meng, Y. T. Su, N. L. Zhou, M. Zhang, M. N. Shao, Y. T. Fan, H. M. Zhu, P. Yuan, C. Chi and Y. H. Xiao, Int. J. Biol. Macromol., 2016, 93, 117-122.
27 C. L. Wang, B. Li, W. F. Niu, S. S. Hong, B. Saif, S. B. Wang, C. Dong and S. M. Shuang, RSC Adv., 2015, 5, 89299-89308. 28 A. Saneja, R. Kumar, D. Arora, S. Kumar, A. K. Panda and S. Jaglan, Drug Discovery Today, 2018, 23, 1115-1125.

29 M. Abdolahad, M. Janmaleki, S. Mohajerzadeh, O. Akhavan and S. Abbasi, Mater. Sci. Eng., C, 2013, 33, 1498-1505.

30 Z. M. Markovic, L. M. Harhaji-Trajkovic, B. M. TodorovicMarkovic, D. P. Kepić, K. M. Arsikin, S. P. Jovanović, A. C. Pantovic, M. D. Dramićanin and V. S. Trajkovic, Biomaterials, 2011, 32, 1121-1129.

31 K. Yang, J. M. Wan, S. Zhang, B. Tian, Y. J. Zhang and Z. Liu, Biomaterials, 2012, 33, 2206-2214.

32 H. Y. Liu, T. Li, Y. H. Liu, G. Q. Qin, X. P. Wang and T. S. Chen, Nanoscale Res. Lett., 2016, 11, 211.

33 W. Zhang, Z. Y. Guo, D. Q. Huang, Z. M. Liu, X. Guo and H. Q. Zhong, Biomaterials, 2011, 32, 8555-8861.

34 B. Tian, C. Wang, S. Zhang, L. Z. Feng and Z. Liu, ACS Nano, 2011, 5, 7000-7009.

35 A. Zuchowska, M. Chudy, A. Dybko and Z. Brzozka, Sens. Actuators, B, 2017, 243, 152-165.

36 L. Zhou, L. Zhou, S. H. Wei, X. F. Ge, J. H. Zhou, H. J. Jiang, F. Y. Li and J. Shen, J. Photochem. Photobiol., B, 2014, 135, 716.

37 Y.-W. Chen, Y.-L. Su, S.-H. Hu and S.-Y. Chen, Adv. Drug Delivery Rev., 2016, 105, 190-204.

38 L. Zhang, Y. C. Li and J. C. Yu, J. Mater. Chem. B, 2014, 2, 452470.

39 J. W. Tian, L. Ding, Q. B. Wang, Y. P. Hu, L. Jia, J.-S. Yu and H. X. Ju, Anal. Chem., 2015, 87, 3841-3848.

40 M. E. Mathew, J. C. Mohan, K. Manzoor, S. V. Nair, H. Tamur and R. Jayakumar, Carbohydr. Polym., 2010, 80, 442-448.

41 E. K. Park, S. B. Lee and Y. M. Lee, Biomaterials, 2005, 26, 1053-1061.

42 X. B. Zhao and P. Liu, RSC Adv., 2014, 4, 24232-24239.

43 D. Depan, J. Shah and R. D. K. Misra, Mater. Sci. Eng., C, 2011, 31, 1305-1312.

44 Q. L. Huang, J. M. Wang, W. X. Wei, Q. X. Yan, C. L. Wu and X. S. Zhu, J. Hazard. Mater., 2015, 283, 123-130.

45 A. Correia, M.-A. Shahbazi, . E. Makilä, S. Almeida, J. Salonen, J. Hirvonen and H. A. Santos, ACS Appl. Mater. Interfaces, 2015, 7, 23197-23204.

46 V. Georgiadou, G. Makris, D. Papagiannopoulou, G. Vourlias and C. Dendrinou-Samara, ACS Appl. Mater. Interfaces, 2016, 8, 9345-9360.

47 D. Li, M. B. Müller, S. Gilje, R. B. Kaner and G. G. Wallace, Nat. Nanotechnol., 2008, 3, 101-105.

48 L. Zhan, Y. Zhang, Q. L. Zeng, Z. D. Liu and C. Z. Huang, J. Colloid Interface Sci., 2014, 426, 293-299.

49 H. Cao, X. Wu, G. Yin and J. H. Warner, Inorg. Chem., 2012, 51, 2954-2960.

50 S. Stankovich, D. A. Dikin, R. D. Piner, K. A. Kohlhaas, A. Kleinhammes, Y. Jia, Y. Wu, S. T. Nguyen and R. S. Ruoff, Carbon, 2007, 45, 1558-1565.

51 J. M. Haider and Z. Pikramenou, Chem. Soc. Rev., 2005, 34, 120-132.

52 A. Kasprzak and M. Poplawska, Chem. Commun., 2018, 54, 8547-8562. 
53 F. Wu, M. Zhang, H. W. Lu, D. Liang, Y. L. Huang, Y. H. Xia, Y. Q. Hu, S. Q. Hu, J. X. Wang, X. Y. Yi and J. Zhang, ACS Appl. Mater. Interfaces, 2018, 10(26), 21939-21949.

54 M. Zhang, F. Wu, W. T. Wang, J. Shen, N. L. Zhou and C. Z. Wu, Chem. Mater., DOI: 10.1021/ acs.chemmater.8b00934.

55 M. Zhang, W. T. Wang, K. Graveran, N.-L. Zhou, J. Zhang and F. Wu, Chem.-Eur. J., 2018, 24, 12890-12901.

56 N. Husain, T. T. Ndou, A. M. de la Peña and I. M. Warnert, Appl. Spectrosc., 1992, 46, 652-658.

57 N. V. Roik and L. A. Belyakova, Interface Focus, 2016, 6, 1-10. 58 K. Nawara, J. Romiszewski, K. Kijewska, J. Szczytko, A. Twardowski, M. Mazur and P. Krysinski, J. Phys. Chem. C, 2012, 116, 5598-5609.
59 Y.-Y. Song, C. Li, X.-Q. Yang, J. An, K. Cheng, Y. Xuan, X.-M. Shi, M.-J. Gao, X.-L. Song, Y.-D. Zhao and W. Chen, J. Mater. Chem. B, 2018, 6, 4808-4820.

60 D. Jaque, L. Martínez Maestro, B. del Rosal, P. HaroGonzalez, A. Benayas, J. L. Plaza, E. Martín Rodríguez and J. García Solé, Nanoscale, 2014, 6, 9494-9530.

61 V. Shanmugam, S. Selvakumar and C.-S. Yeh, Chem. Soc. Rev., 2014, 43, 6254-6287.

62 T. Li, H. Y. Liu, G. N. Xi, Y. L. Pang, L. P. Wu, X. P. Wang and T. S. Chen, J. Mater. Chem. B, 2016, 4, 2972-2983.

63 H. D. Chen, F. Y. Liu, Z. Lei, L. N. Ma and Z. X. Wang, RSC Adv., 2015, 5, 84980-84987. 